\title{
Entropy as Measure of Brain Networks' Complexity in Eyes Open and Closed Conditions
}

\author{
Fabrizio Vecchio ${ }^{1,2, * \mathbb{D}}$, Francesca Miraglia ${ }^{1}$, Chiara Pappalettera ${ }^{1} \mathbb{D}$, Alessandro Orticoni ${ }^{1}$, Francesca Alù ${ }^{1}$, \\ Elda Judica ${ }^{3}$, Maria Cotelli ${ }^{4}$ and Paolo Maria Rossini ${ }^{1}$ \\ 1 Brain Connectivity Laboratory, Department of Neuroscience and Neurorehabilitation, IRCCS San Raffaele \\ Roma, 00166 Rome, Italy; fra.miraglia@gmail.com (F.M.); pappaletterachiara@gmail.com (C.P.); \\ alessandro.orticoni@gmail.com (A.O.); alufrancesca@yahoo.it (F.A.); paolomaria.rossini@sanraffele.it (P.M.R.) \\ 2 Department of Theoretical and Applied Sciences, eCampus University, 22060 Novedrate, Italy \\ 3 Department of Neurorehabilitation Sciences, Casa Cura Policlinico, 20144 Milano, Italy; e.judica@ccppdezza.it \\ 4 Neuropsychology Unit, IRCCS Istituto Centro San Giovanni di Dio Fatebenefratelli, 25125 Brescia, Italy; \\ mcotelli@fatebenefratelli.eu \\ * Correspondence: fabrizio.vecchio@uniecampus.it or fabrizio.vecchio@sanraffaele.it; Tel.: +39-06-52253767
}

Citation: Vecchio, F.; Miraglia, F.; Pappalettera, C.; Orticoni, A.; Alù, F.; Judica, E.; Cotelli, M.; Rossini, P.M. Entropy as Measure of Brain Networks' Complexity in Eyes Open and Closed Conditions. Symmetry 2021, 13, 2178. https://doi.org/ $10.3390 /$ sym 13112178

Academic Editor: Giorgio Vallortigara

Received: 18 June 2021

Accepted: 10 November 2021

Published: 15 November 2021

Publisher's Note: MDPI stays neutral with regard to jurisdictional claims in published maps and institutional affiliations.

\begin{abstract}
Brain complexity can be revealed even through a comparison between two trivial conditions, such as eyes open and eyes closed (EO and EC respectively) during resting. Electroencephalogram (EEG) has been widely used to investigate brain networks, and several non-linear approaches have been applied to investigate EO and EC signals modulation, both symmetric and not. Entropy is one of the approaches used to evaluate the system disorder. This study explores the differences in the EO and EC awake brain dynamics by measuring entropy. In particular, an approximate entropy (ApEn) was measured, focusing on the specific cerebral areas (frontal, central, parietal, occipital, temporal) on EEG data of 37 adult healthy subjects while resting. Each participant was submitted to an EO and an EC resting EEG recording in two separate sessions. The results showed that in the EO condition the cerebral networks of the subjects are characterized by higher values of entropy than in the EC condition. All the cerebral regions are subjected to this chaotic behavior, symmetrically in both hemispheres, proving the complexity of networks dynamics dependence from the subject brain state. Remarkable dynamics regarding cerebral networks during simple resting and awake brain states are shown by entropy. The application of this parameter can be also extended to neurological conditions, to establish and monitor personalized rehabilitation treatments.
\end{abstract}

Keywords: entropy; eyes; EEG; brain network

\section{Introduction}

The first electroencephalographic (EEG) recording was performed in 1924 by the German neurologist Hans Berger, the inventor of this brain electrical activity recording technique. He demonstrated that a comparison between two apparently trivial conditions, such as eyes open and eyes closed (EO and EC respectively) while resting, can reveal the extent of human brain complexity; in particular he discovered that when a healthy subject opens the eyes, the amplitude of alpha waves $(8-13 \mathrm{~Hz})$ decreases (the so-called alpha blockage) [1]. Since Berger published the first paper about scalp EEG, this technique has been widely applied to the study of the brain [2], both with the linear and nonlinear approach. His findings have been confirmed by several following theories: these demonstrate that alpha desynchronization (due to the visual input in EO condition) reveals the increment of functional innervation of the visual system, which leads to an activation of a larger area of cortex and reflects the interactions of cortical and thalamo-cortical systems that aid the information flow [3-5]. Several studies extended the observations to different bands of frequency: reductions have been found in the cerebral activity power from the EC to EO condition, both in young and older subjects, suggesting a preservation of the energetics of EEG reactivity in healthy aging [6]. 
Besides the power spectrum analysis, the two conditions and the related cerebral networks changes have been explored through brain connectivity techniques, namely the evaluation of the functional interactions among the different brain areas. In particular, a mathematical description of the cerebral architecture, the graph theory, is employed to analyze EEG data for the investigation of the neural network organization [7]. The small-world value, a parameter explaining the balance between brain networks local specialization and global integration [8,9], has been found as higher in alpha band and lower in beta2 and gamma $(20-30 \mathrm{~Hz}$ and $30-45 \mathrm{~Hz}$ respectively) bands in the resting EO condition with respect to the EC one, in healthy elderly subjects [10]. The same applies to healthy young adults, in which the small-world value increases from EC to EO states in the alpha band and reduces slightly both in the theta and in the beta (4-7 Hz and $14-30 \mathrm{~Hz}$ respectively) band [11].

Even non-linear approaches have been extensively employed to explore the physiological mechanisms of cerebral functioning [12]: in fact, a dynamic and complex system such as the human brain is mostly characterized by transient and time-varying interaction phenomena among the topographically distributed neural networks, both symmetrically and asymmetrically. In particular, non-linear approaches have been used to explore the differences in terms of brain functional configuration between EO and EC conditions [13-15]. One of these approaches is represented by the chaotic statistics. Specifically, the brain chaotic behavior is explored through the entropy, a characteristic defined as the measurement of the changing complexity and disorder in any system; less ordered and more random systems are typically associated with higher entropy values.

Brain activity is studied by means of many entropy parameters starting from the hypothesis of the entropic brain that can be characterized by uncertainty and information contents [16]. Different works have investigated the differences between EO and EC resting state conditions. The Acceleration Spectrum Entropy (ASEn) has been found to increase significantly all over the scalp from the EC to EO condition, proving to be sensitive to desynchronization [17]. Other entropy indices, such as the Sample Entropy (SampEn) and the Normalized Corrected Shannon Entropy (NCSEn), have been shown to be higher in the EO than in the EC condition, the former in frontal, temporal, and parietal cerebral areas, and the latter over all the cortex, suggesting that entropy parameters are sensitive to increases during external stimuli processing $[13,18]$. One of the most used indices of the entropy in brain activity examination [19-22] is the approximate entropy (ApEn). It is known for the advantages related to its properties: it maintains a good reproducibility if used with the time series of at least 50 samples; it is mostly insensitive to noise; it is finite for composite, stochastic, and noisy deterministic processes [23]; and it reveals the underlying episodic behavior changes undetected by peak occurrences or amplitudes [22]. Within this theoretical framework, the aim of this study is to investigate the differences and the symmetry in the brain dynamics of healthy subjects in the passage from the EO to EC conditions by means of entropy applying ApEn parameter, as the ApEn would be very useful to understand brain complexity and dynamical characteristics.

\section{Materials and Methods}

\subsection{Participants}

A group of 37 healthy volunteers was recruited (18 females; age $=26.0 \pm 0.5$ (Mean \pm Standard Error -SE-) years of age, education $=16.3 \pm 0.3$ (Mean \pm SE) years), whose demographic data are shown in Table 1 . The exclusion criteria consisted of current therapy with psychotropic or vasoactive medication, and psychiatric or neurological disorder history. All subjects were right-handed at the Handedness Questionnaire [24]. Participants were subjected to an EO and an EC EEG recording in two separate resting sessions of about 6 min each, for a total of 74 recordings. All experiments were undertaken after obtaining the informed and signed consent of all participants, in agreement to the Code of Ethics of the World Medical Association (1997), and the experimental methods were compliant with the Declaration of Helsinki. The EEG protocol was performed in accordance with 
safety guidelines procedures. To avoid possible behavioral effects, before starting the EEG recording the participants were instructed to stay as relaxed as possible and to avoid movements. During the eyes open condition they were asked to look at a fixed point in front of them in order to avoid the orienting network activation due to the visual stimulus selection operated by the subject.

Table 1. Sex, age and education data of all subjects enrolled in the experiment.

\begin{tabular}{cc}
\hline & Participants $(\mathbf{n}=\mathbf{3 7})$ \\
\hline Sex $(\mathrm{M} / \mathrm{F})$ & $19 / 18$ \\
Age $($ mean $\pm S E)$ & $26 \pm 0.5$ \\
Education $($ mean $\pm S E)$ & $16 \pm 0.3$ \\
\hline
\end{tabular}

\subsection{Data Recordings and Preprocessing}

A digital EEG device, BrainAmp (by Brain Products), was employed to carry out the EEG recordings, from 64 channels, placed following the International 10-20 system with the reference electrode positioned in FCz. In order to monitor eyes blinking, both vertical and horizontal EOGs were used. The sampling rate frequency was set up at $1000 \mathrm{~Hz}$ and the impedance of the electrodes was maintaned below $5 \mathrm{~K} \Omega$. EEG activity was assessed at rest, without any task to perform, in at least 6 min of eyes-closed and 6 min of eyes-open (EC and EO respectively) conditions, avoiding blinking, and staring at a fixation point without shifting the gaze during the EO condition. During the examination, participants were located in a sound-damped, dimly lit, and electrically shielded room and seated in a comfortable armchair. The Matlab software (MathWorks, Natick, MA, USA) was employed in order to process the data, using codes built on the EEGLAB toolbox (Swartz Center for Computational Neurosciences, La Jolla, CA, USA) $[25,26]$. The EEG signals were downsampled at $512 \mathrm{~Hz}$ and filtered from 0.2 to $47 \mathrm{~Hz}$ using a band-pass finite impulse response filter (FIR). A duration of $2 \mathrm{~s}$ epochs were extracted from continuous imported data, and EEG signals principal artifacts (i.e., scalp muscle contraction, eye movements and cardiac activity) were removed first by an EEG expert visual inspection, and later through the Infomax ICA algorithm [27], that allows the division of sources statistically independent from each other [28-31], as realized in the EEGLAB. After the cleaning procedure, at least 5 min remained for each session.

\subsection{Entropy Analysis}

An analysis of entropy was carried out on the epochs cleaned from the artifacts using a home-fabricated software created in Matlab. The software core computed the ApEn estimation using the algorithm implemented in Matlab and specifying two input parameters: a tolerance factor, $r$, and a pattern length, $\mathrm{m}$, to compute it. $r$ is also named as a similarity factor used to detect a similarity range between data points. The ApEn values generated by the software are unitless and range from 0 to 2 , where 0 corresponds to a regular time series, and 2 to a random time series [32]. Summarizing, the calculation of ApEn works as follows:

1. A point by point comparison between every $m$-long data sequence and all the other sequences are performed, and when all points are within $r$, a match is counted.

2. The same process is performed on the next sequences of length $m+1$, starting with the $m+1$ points first sequence.

3. The matches number is turned into a natural logarithm value, and later the normalization with regard to the data points number $(N)$ is implemented.

The ApEn is calculated as $A p E n=\Phi_{m}-\Phi_{m+1}$, where:

$$
\phi_{m}=(N-m+1)^{-1} \sum_{i=1}^{N-m+1} \log \left(N_{i}\right)
$$


Moreover, $N_{i}$ represents the amount of points that are within $r$ of their corresponding point in the original sequence $Y$, at the point $i$ :

$$
N_{i}=\sum_{i=1, i \neq k}^{N}\left(\left\|Y_{i}-Y_{k}\right\|_{\infty}<r\right)
$$

In the present analysis, the Matlab default values for $\mathrm{m}$ and $\mathrm{r}$ were utilized: $\mathrm{so}$, $\mathrm{m}$ was equal to 2 and $r$ to $0.2 *$ variance (x) [33-35], with $x$ that corresponds to a $2 \mathrm{~s}$ long epoch of a specific channel. A low ApEn of the investigated signal is suggestive of a high similarity between data sequences [19]. The values of ApEn calculated on each single epoch were averaged to obtain a value for each channel [36]. The ApEn values computed in each channel were thus averaged over ten regions of interest (ROIs), five for each hemisphere (frontal, central, parietal, occipital, temporal) $[19,20]$, in order to obtain a single value of entropy for each ROI, as shown in Figure 1. The midline channels were not considered. Taking into account the modalities of evaluation of the ApEn, it may result as challenging to visually detect the differences in terms of complexity traced by the EEG.

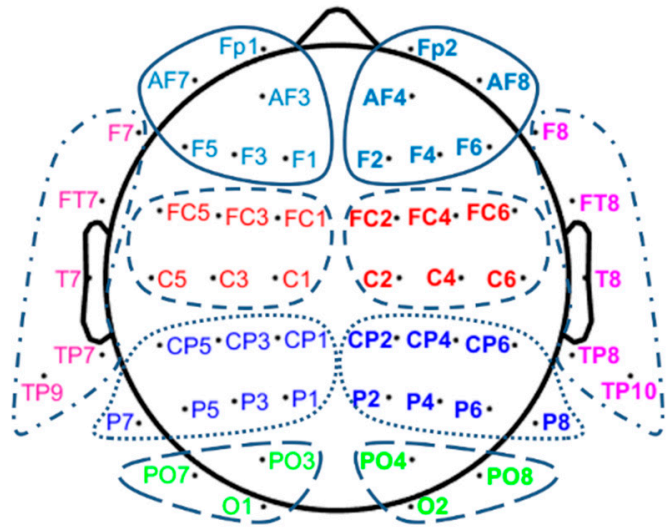

\begin{tabular}{|c|c|c|c|c|}
\hline $\begin{array}{c}\begin{array}{c}\text { Frontal } \\
\text { Left }\end{array} \\
\end{array}$ & $\left.\mid \begin{array}{cc}1 & \text { Central } \\
1 & 1 \\
1 & \text { Left__ }\end{array}\right]$ & $\begin{array}{c}\text { Parietal } \\
\text { Left } \\
\end{array}$ & $\mid \begin{array}{l}\text { Occipital } \\
\text { 는 Left }\end{array}$ & Temporal \\
\hline $\mathrm{FP1}$ & $\mathrm{FC1}$ & CP1 & PO3 & F7 \\
\hline $\mathrm{AF} 3$ & $\mathrm{FC} 3$ & $\mathrm{CP} 3$ & PO7 & FT7 \\
\hline Af7 & FC5 & CP5 & 01 & $\mathrm{T7}$ \\
\hline $\mathrm{F} 1$ & $\mathrm{C} 1$ & P1 & & TP7 \\
\hline $\mathrm{F} 3$ & C3 & P3 & & TP9 \\
\hline \multirow[t]{2}{*}{ F5 } & C5 & P5 & & \\
\hline & & P7 & & \\
\hline $\begin{array}{c}\text { Frontal } \\
\text { Right }\end{array}$ & $\mid \begin{array}{lll}\text { Central } \\
1 \\
1\end{array}$ & $\begin{array}{l}\text { Parietal } \\
\text { Right }\end{array}$ & Occipital & Temporal \\
\hline FP2 & $\mathrm{FC2}$ & CP2 & $\mathrm{PO4}$ & F8 \\
\hline AF4 & FC4 & CP4 & P08 & FT8 \\
\hline AF8 & FC6 & CP6 & 02 & T8 \\
\hline$F 2$ & C2 & P2 & & TP8 \\
\hline F4 & C4 & P4 & & TP10 \\
\hline \multirow[t]{2}{*}{ F6 } & C6 & P6 & & \\
\hline & & P8 & & \\
\hline
\end{tabular}

Figure 1. Left panel: clustering of the channels according to regions of interest (ROIs of the brain networks), regular font for left, bold font for right hemisphere: solid and light blue for frontal, dashed and red for central, dotted and blue for parietal, long-dashed and green for occipital, dash-dotted line and magenta color for temporal network. Right: table of the channels according to regions of interest (ROIs).

\subsection{Statistical Evaluation}

The analysis of variance (ANOVA) was employed to evaluate ApEn values calculated in the five ROIs for both the cerebral hemispheres for all the recordings. In order to avoid a possible violation of the sphericity assumption in the repeated ANOVA, the Geisser and Greenhouse correction was used. Moreover, the post-hoc analysis was performed by means of the Duncan's test and 0.05 significance level.

A three-way ANOVA was performed among the factors: condition (EO, EC, between values), side (left, right), and ROI (frontal, central, parietal, occipital, temporal).

\section{Results}

The ANOVA for the ApEn evaluation exhibited a statistically significant main effect of Condition $(\mathrm{F}(1,36)=36.120, p=0.0000)$, meaning that the brain networks are characterized by higher values of entropy while awake subjects voluntarily keep their eyes open rather than closed, regardless of the brain region and the hemisphere.

Furthermore, the ANOVA exhibited a statistically significant interaction $(\mathrm{F}(4,144)=4.7992$, $p=0.0012$ ) among the factors Condition (EO, EC) and ROI (frontal, central, parietal, occipital, temporal) as shown in Figure 2. The Duncan post-hoc testing $(p<0.001)$ revealed higher entropy values in the frontal, central, parietal, occipital and temporal ROIs during the EO 
condition compared with the EC one, thus for all the investigated brain areas. No statistically significant interaction among the factors condition and hemisphere was found.

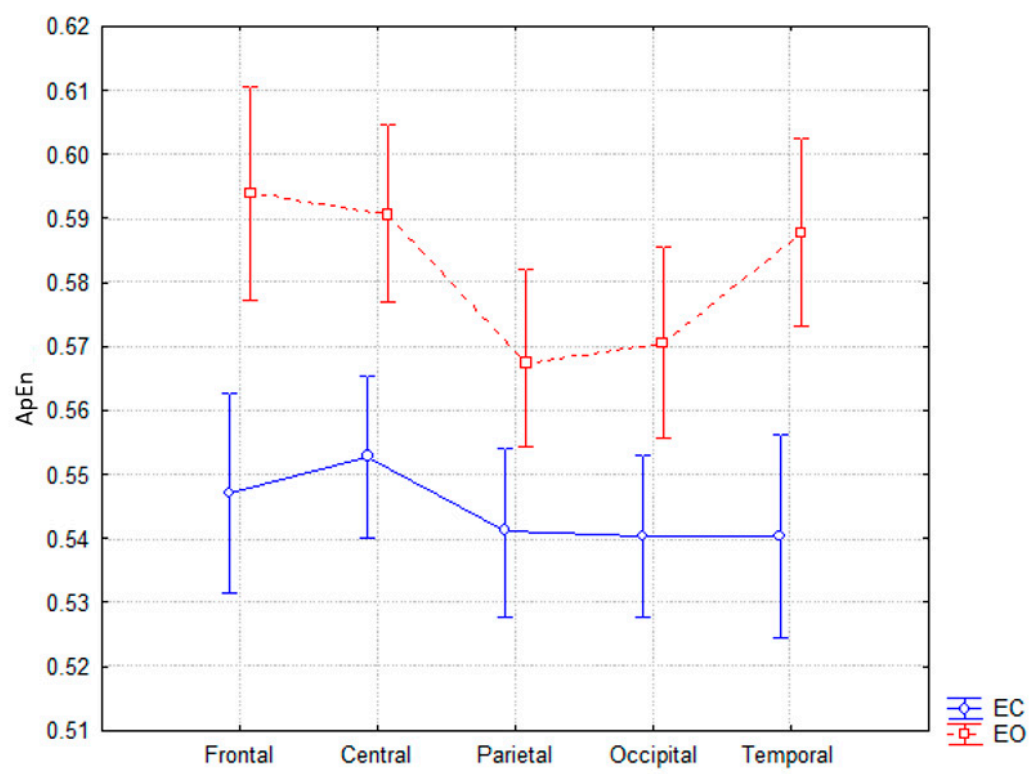

Figure 2. Statistically significant interaction of $\operatorname{ApEn}(F(4,144)=4.7992, p=0.0012)$ among the factors condition (EO, EC) and ROI (frontal, central, parietal, occipital, temporal).

\section{Discussion}

This study aimed to investigate how the cerebral dynamics are modulated by the effect of a simple condition such as keeping the eyes open or closed; this chaotic behavior (of the brain) was revealed using the entropy non-linear parameter, the approximate entropy (ApEn), applied to EEG recordings in resting condition. The current study expands previous studies $[13,17,18,37]$ investigating the complexity of brain activity in various areas and seeking eventual symmetric effects between hemispheres. To the best of our knowledge, with respect to the current literature, the main novel finding of the present study is the computation of ApEn selecting 10 cerebral networks, 5 in each brain hemispheres, to explore the effects of eyes open or closed information processes on EEG activity. Thus, the computation of ApEn in 10 brain ROIs by grouping 64 electrodes in the frontal, central, parietal, occipital and temporal areas for each hemisphere, allowed us to be as focal as possible, as well as more specific in the investigation of possible differences across the entire brain.

The current results revealed that a higher entropy characterizes human brain networks while subjects keep their eyes open. Compared with previous studies, we conducted a more detailed research into the entire brain, dividing it into focal regions. Even though the result does not particularly help us to explain our novel finding, we can dissert that there is not a specific region or hemisphere showing a different behavior from the entire brain between the two brain states studied (eyes open and eyes closed). In fact, this chaotic behavior of the brain emerges in all the explored cerebral regions and symmetrically in both the hemispheres, thus confirming the complexity of brain networks dynamics, which clearly changes while switching between two apparently trivial brain states. Furthermore, no differences were found between the two hemispheres with respect to the two resting state conditions: this suggests that the modulation due to the opening of the eyes affects symmetrically both hemispheres.

Eyes closed and eyes open statuses are widely studied resting conditions: they can be discriminated by the computation of mathematical features [38]. Compared with the eyes closed state, during the eyes open state the brain is stressed with a higher number of external stimuli resulting, from a functional perspective, in a state of involuntary information 
processing and mental activity, although still at rest [39,40]. Consequently, the high entropy values are indicative of an increase in the EEG signal irregularity that characterizes the desynchronization of brain activity [17]. Entropy measures the predictability of a signal, thus a higher degree of randomness distributed all over the channels, even if considered separately, describes a reduction in brain synchronization of the neural networks' oscillatory activity: this activity represents the electrophysiological correlation of increased cortical excitability and brain generalized arousal [41,42]. A study applying transfer entropy to resting EEG recordings showed that the information that flows into the brain is greater during the EC than EO state, mainly from the posterior to the frontal lobe [43]. This finding supports the hypothesis that while keeping the eyes closed, the brain is mostly involved in internal processes rather than in external stimuli ones, resulting in an increase in cerebral synchronization. Summarizing, the desynchronized EEG activity reflects an information processing and mental activity state that particularly characterizes the EO condition compared with EC.

Numerous studies have suggested that brain activity desynchronization reflects the increased functions of the visual system caused by visual stimulus, which is mediated by the reticular activating system. This process involves different structural components resulting in a complex dynamic in the EO condition compared with the EC one [44,45]. Due to the distributed organization of cortical networks involved in the vision, the representation and the perception of visual objects requires the integration of responses across different brain regions [46]. In particular, the occipital and temporal areas play a crucial role in information receiving and objects' recognition [47], while the frontal ones are engaged in generating visual perception contents and supporting the perception of learned objects, events, and actions [48,49].

The visual information processing can be modulated by the attention paid by the subject to the surroundings, which may cause far away effects upon the visual cortex wielded by cerebral structures outside of the visual system [50]. The process of orienting to sensory objects, namely picking out information from sensory input, is called orienting attention. The orienting network, particularly for visual events, relies heavily on frontal eye fields and on parietal systems, comprising the temporal parietal junction and the superior parietal lobe [51]; the basal forebrain with its cholinergic systems also seems to play a fundamental role in controlling attentional orienting [52].

Based on the research, supposedly even during resting (state) the subject, sitting with the eyes open during the EEG recording, tends to pay attention to the surroundings, thus to all the available visual inputs.

In conclusion, the visual information processing and the attention paid to the visual environment, although involuntary, correlate with a symmetric EEG desynchronization spread almost over the whole brain, as revealed by the high entropy values in all the investigated cortical regions.

\section{Limitations}

Some potential methodology limitations of this study should be taken into account. Each EEG experiment is intrinsically affected by the volume conduction error. In order to solve this issue, in the current study we computed the common average of the data. Moreover, although the electrodes cannot be placed in the same fixed position for all the subjects, we can perform such an approximation. In fact, we computed the value of ApEn in each ROI as the average of the ApEn values of each electrode. Accordingly, the localization error was minimized as the values of the ApEn come from a set of averaged electrodes representative of a larger brain area and not only from a single electrode. For these reasons, we believe that the localization error across different subjects is minimized. Furthermore, the computation related to ApEn is limited to a partial knowledge of input parameters' values, using the default parameter of the Matlab function, although it is widely used in the literature. Remarkably, future studies should investigate how the variation in this parameter can affect the results. 


\section{Conclusions}

In conclusion, the EO condition is characterized by higher values of entropy all throughout the brain compared with the EC one. This finding reflects the EEG desynchronization due to visual information receiving and processing, and to the attention of the subject to the surrounding. The results emphasize the interesting dynamics in the cerebral networks as revealed by the entropy parameter during resting conditions; in fact, entropy indices, including the one investigated in the present study, seem to be sensitive to information processing demands in all the ROIs considered and to be symmetric to the hemispheres. In future studies it would be interesting to employ this parameter to explore neurological and psychiatric disorders (i.e., Parkinson's disease, Alzheimer's disease, schizophrenia, Sleep Apnea, etc.) in order to detect biomarkers for an early diagnosis, as well as for monitoring the impact of eventual personalized pharmacological and rehabilitation treatments.

Author Contributions: Conceptualization, F.M. and F.V.; methodology, F.M., C.P., F.A., E.J., M.C. and F.V.; software, A.O. and F.V.; validation, A.O.; data curation, F.A.; writing-original draft preparation, F.V. and A.O.; writing-review and editing, C.P., F.A., E.J., M.C., P.M.R. and F.M.; supervision, F.M. All authors have read and agreed to the published version of the manuscript.

Funding: This research received no external funding.

Institutional Review Board Statement: The study was conducted according to the guidelines of the Declaration of Helsinki. Ethical review and approval were waived for this study, because it was conducted within IRCCS (scientific hospitalization and treatment institutes).

Informed Consent Statement: Informed consent was obtained from all subjects involved in the study.

Data Availability Statement: The data that support the findings of this study are available on request from the corresponding author.

Acknowledgments: This work was partially supported by the Italian Ministry of Health for Institutional Research (Ricerca corrente).

Conflicts of Interest: The authors declare no conflict of interest.

\section{References}

1. Kirschfeld, K. The physical basis of alpha waves in the electroencephalogram and the origin of the "Berger effect". Biol. Cybern. 2005, 92, 177-185. [CrossRef] [PubMed]

2. Millett, D. Hans Berger: From psychic energy to the EEG. Perspect. Biol. Med. 2001, 44, 522-542. [CrossRef] [PubMed]

3. Kan, D.P.X.; Croarkin, P.E.; Phang, C.K.; Lee, P.F. EEG Differences Between Eyes-Closed and Eyes-Open Conditions at the Resting Stage for Euthymic Participants. Neurophysiology 2017, 49, 432-440. [CrossRef]

4. Barry, R.J.; Clarke, A.R.; Johnstone, S.J.; Magee, C.A.; Rushby, J.A. EEG differences between eyes-closed and eyes-open resting conditions. Clin. Neurophysiol. 2007, 118, 2765-2773. [CrossRef]

5. Mediano, P.A.M.; Rosas, F.E.; Timmermann, C.; Roseman, L.; Nutt, D.J.; Feilding, A.; Kaelen, M.; Kringelbach, M.L.; Barrett, A.B.; Seth, A.K.; et al. Carhart-Harris RL: Effects of external stimulation on psychedelic state neurodynamics. bioRxiv 2020. [CrossRef]

6. Barry, R.J.; De Blasio, F.M. EEG differences between eyes-closed and eyes-open resting remain in healthy ageing. Biol. Psychol. 2017, 129, 293-304. [CrossRef]

7. Vecchio, F.; Miraglia, F.; Maria Rossini, P. Connectome: Graph theory application in functional brain network architecture. Clin Neurophysiol. Pract. 2017, 2, 206-213. [CrossRef]

8. Vecchio, F.; Miraglia, F.; Bramanti, P.; Rossini, P.M. Human brain networks in physiological aging: A graph theoretical analysis of cortical connectivity from EEG data. J. Alzheimer's Dis. 2014, 41, 1239-1249. [CrossRef] [PubMed]

9. Vecchio, F.; Miraglia, F.; Piludu, F.; Granata, G.; Romanello, R.; Caulo, M.; Onofrj, V.; Bramanti, P.; Colosimo, C.; Rossini, P.M. "Small World" architecture in brain connectivity and hippocampal volume in Alzheimer's disease: A study via graph theory from EEG data. Brain Imaging Behav. 2017, 11, 473-485. [CrossRef]

10. Miraglia, F.; Vecchio, F.; Bramanti, P.; Rossini, P.M. EEG characteristics in "eyes-open" versus "eyes-closed" conditions: Smallworld network architecture in healthy aging and age-related brain degeneration. Clin. Neurophysiol. 2016, 127, 1261-1268. [CrossRef]

11. Tan, B.; Kong, X.; Yang, P.; Jin, Z.; Li, L. The difference of brain functional connectivity between eyes-closed and eyes-open using graph theoretical analysis. Comput. Math. Methods Med. 2013, 2013, 976365. [CrossRef] [PubMed] 
12. Ma, Y.; Shi, W.; Peng, C.K.; Yang, A.C. Nonlinear dynamical analysis of sleep electroencephalography using fractal and entropy approaches. Sleep Med. Rev. 2018, 37, 85-93. [CrossRef] [PubMed]

13. Hogan, M.J.; Kilmartin, L.; Keane, M.; Collins, P.; Staff, R.T.; Kaiser, J.; Lai, R.; Upton, N. Electrophysiological entropy in younger adults, older controls and older cognitively declined adults. Brain Res. 2012, 1445, 1-10. [CrossRef] [PubMed]

14. Blackburn, D.J.; Zhao, Y.; De Marco, M.; Bell, S.M.; He, F.; Wei, H.L.; Lawrence, S.; Unwin, Z.C.; Blyth, M.; Angel, J.; et al. A Pilot Study Investigating a Novel Non-Linear Measure of Eyes Open versus Eyes Closed EEG Synchronization in People with Alzheimer's Disease and Healthy Controls. Brain Sci. 2018, 8, 134. [CrossRef]

15. Díaz, H.M.; Maureira, F.; Cordova, F. Time series of closed and open eyes EEG conditions reveal differential characteristics in the temporality of linear and non-linear analysis domain. Procedia Comput. Sci. 2018, 139, 570-577.

16. Carhart-Harris, R.L. The entropic brain-revisited. Neuropharmacology 2018, 142, 167-178. [CrossRef]

17. Stam, C.J.; Tavy, D.L.; Keunen, R.W. Quantification of alpha rhythm desynchronization using the acceleration spectrum entropy of the EEG. Clin. Electroencephalogr. 1993, 24, 104-109. [CrossRef]

18. Hussain, L.; Aziz, W.; Alowibdi, J.S.; Habib, N.; Rafique, M.; Saeed, S.; Kazmi, S.Z. Symbolic time series analysis of electroencephalographic (EEG) epileptic seizure and brain dynamics with eye-open and eye-closed subjects during resting states. J. Physiol. Anthropol. 2017, 36, 21. [CrossRef]

19. Alù, F.; Miraglia, F.; Orticoni, A.; Judica, E.; Cotelli, M.; Rossini, P.M.; Vecchio, F. Approximate Entropy of Brain Network in the Study of Hemispheric Differences. Entropy 2020, 22, 1220. [CrossRef]

20. Alù, F.; Orticoni, A.; Judica, E.; Cotelli, M.; Rossini, P.; Miraglia, F.; Vecchio, F. Entropy modulation of brain electroencephalographic signals in physiological aging. Mech. Ageing Dev. 2021, 196, 111472. [CrossRef]

21. Posener, J.A.; Charles DeBattista Veldhuis, J.D.; Province, M.A.; Williams, G.H.; Schatzberg, A.F. Process irregularity of cortisol and adrenocorticotropin secretion in men with major depressive disorder. Psychoneuroendocrinology 2004, 29, 1129-1137. [CrossRef]

22. Pincus, S.M.; Viscarello, R.R. Approximate entropy: A regularity measure for fetal heart rate analysis. Obstet. Gynecol. 1992, 79, 249-255.

23. Pincus, S.M. Assessing serial irregularity and its implications for health. Ann. N. Y. Acad. Sci. 2001, 954, 245-267. [CrossRef] [PubMed]

24. Salmaso, D.; Longoni, A.M. Problems in the assessment of hand preference. Cortex 1985, 21, 533-549. [CrossRef]

25. Vecchio, F.; Miraglia, F.; Judica, E.; Cotelli, M.; Alù, F.; Rossini, P.M. Human brain networks: A graph theoretical analysis of cortical connectivity normative database from EEG data in healthy elderly subjects. GeroScience 2020, 42, 575. [CrossRef]

26. Miraglia, F.; Vecchio, F.; Bramanti, P.; Rossini, P.M. Small-worldness characteristics and its gender relation in specific hemispheric networks. Neuroscience 2015, 310, 1-11. [CrossRef] [PubMed]

27. Miraglia, F.; Vecchio, F.; Marra, C.; Quaranta, D.; Alù, F.; Peroni, B.; Granata, G.; Judica, E.; Cotelli, M.; Rossini, P.M. Small World Index in Default Mode Network Predicts Progression from Mild Cognitive Impairment to Dementia. Int. J. Neural. Syst. 2020, 30, 2050004. [CrossRef]

28. Vecchio, F.; Miraglia, F.; Quaranta, D.; Lacidogna, G.; Marra, C.; Rossini, P.M. Learning Processes and Brain Connectivity in A Cognitive-Motor Task in Neurodegeneration: Evidence from EEG Network Analysis. J. Alzheimer's Dis. 2018, 66, 471-481. [CrossRef]

29. Hoffmann, S.; Falkenstein, M. The correction of eye blink artefacts in the EEG: A comparison of two prominent methods. PLoS ONE 2008, 3, e3004. [CrossRef]

30. Iriarte, J.; Urrestarazu, E.; Valencia, M.; Alegre, M.; Malanda, A.; Viteri, C.; Artieda, J. Independent component analysis as a tool to eliminate artifacts in EEG: A quantitative study. J. Clin. Neurophysiol. 2003, 20, 249-257. [CrossRef]

31. Jung, T.P.; Makeig, S.; Humphries, C.; Lee, T.W.; McKeown, M.J.; Iragui, V.; Sejnowski, T.J. Removing electroencephalographic artifacts by blind source separation. Psychophysiology 2000, 37, 163-178. [CrossRef]

32. Montesinos, L.; Castaldo, R.; Pecchia, L. On the use of approximate entropy and sample entropy with centre of pressure time-series. J. Neuroeng. Rehabil. 2018, 15, 116. [CrossRef] [PubMed]

33. Lee, G.M.; Fattinger, S.; Mouthon, A.L.; Noirhomme, Q.; Huber, R. Electroencephalogram approximate entropy influenced by both age and sleep. Front. Neuroinform. 2013, 7, 33. [CrossRef] [PubMed]

34. Abásolo, D.; Hornero, R.; Espino, P.; Poza, J.; Sánchez, C.I.; de la Rosa, R. Analysis of regularity in the EEG background activity of Alzheimer's disease patients with Approximate Entropy. Clin. Neurophysiol. 2005, 116, 1826-1834. [CrossRef] [PubMed]

35. Burioka, N.; Miyata, M.; Cornélissen, G.; Halberg, F.; Takeshima, T.; Kaplan, D.T.; Suyama, H.; Endo, M.; Maegaki, Y.; Nomura, T.; et al. Approximate entropy in the electroencephalogram during wake and sleep. Clin. EEG Neurosci. 2005, 36, 21-24. [CrossRef]

36. Sun, R.; Wong, W.W.; Wang, J.; Tong, R.K. Changes in Electroencephalography Complexity using a Brain Computer InterfaceMotor Observation Training in Chronic Stroke Patients: A Fuzzy Approximate Entropy Analysis. Front. Hum. Neurosci. 2017, 11, 444. [CrossRef]

37. Olejarczyk, E.; Marzetti, L.; Pizzella, V.; Zappasodi, F. Comparison of connectivity analyses for resting state EEG data. J. Neural. Eng. 2017, 14, 036017. [CrossRef]

38. Mohamed, A.M.A.; Uçan, O.N.; Bayat, O.; Duru, A.D. Classification of Resting-State Status Based on Sample Entropy and Power Spectrum of Electroencephalography (EEG). Appl. Bionics Biomech. 2020, 2020, 8853238. [CrossRef] 
39. Wei, J.; Chen, T.; Li, C.; Liu, G.; Qiu, J.; Wei, D. Eyes-Open and Eyes-Closed Resting States With Opposite Brain Activity in Sensorimotor and Occipital Regions: Multidimensional Evidences From Machine Learning Perspective. Front. Hum. Neurosci. 2018, 12, 422. [CrossRef]

40. Marx, E.; Stephan, T.; Nolte, A.; Deutschländer, A.; Seelos, K.C.; Dieterich, M.; Brandt, T. Eye closure in darkness animates sensory systems. Neuroimage 2003, 19, 924-934. [CrossRef]

41. Daly, I.; Blanchard, C.; Holmes, N.P. Cortical excitability correlates with the event-related desynchronization during braincomputer interface control. J. Neural. Eng. 2018, 15, 026022. [CrossRef]

42. Aono, K.; Miyashita, S.; Fujiwara, Y.; Kodama, M.; Hanayama, K.; Masakado, Y.; Ushiba, J. Relationship between event-related desynchronization and cortical excitability in healthy subjects and stroke patients. Tokai J. Exp. Clin. Med. 2013, 38, 123-128. [PubMed]

43. Olejarczyk, E.; Zuchowicz, U.; Wozniak-Kwasniewska, A.; Kaminski, M.; Szekely, D.; David, O. The Impact of Repetitive Transcranial Magnetic Stimulation on Functional Connectivity in Major Depressive Disorder and Bipolar Disorder Evaluated by Directed Transfer Function and Indices Based on Graph Theory. Int. J. Neural. Syst. 2020, 30, 2050015. [CrossRef] [PubMed]

44. Volavka, J.; Matousek, M.; Roubícek, J. Mental arithmetic and eye opening. An EEG frequency analysis and GSR study. Electroencephalogr. Clin. Neurophysiol. 1967, 22, 174-176. [CrossRef]

45. Gale, A.; Coles, M.; Boyd, E. Variation in visual input and the occipital EEG: II. Psychon. Sci. 1971, 23, 99-100. [CrossRef]

46. Uhlhaas, P.J.; Singer, W. Neural Synchrony in Brain Disorders: Relevance for Cognitive Dysfunctions and Pathophysiology. Neuron 2006, 52, 155-168. [CrossRef]

47. Kastner, S.; Ungerleider, L.G. Mechanisms of visual attention in the human cortex. Annu. Rev. Neurosci. 2000, $23,315-341$. [PubMed]

48. Libedinsky, C.; Livingstone, M. Role of prefrontal cortex in conscious visual perception. J. Neurosci. 2011, 31, 64-69. [CrossRef]

49. Schall, J.D. Visuomotor Functions in the Frontal Lobe. Annu. Rev. Vis. Sci. 2015, 1, 469-498. [CrossRef]

50. Vuilleumier, P.; Driver, J. Modulation of visual processing by attention and emotion: Windows on causal interactions between human brain regions. Philos. Trans. R. Soc. B Biol. Sci. 2007, 362, 837-855. [CrossRef] [PubMed]

51. Raz, A. Anatomy of attentional networks. Anat. Rec. Part B N. Anat. 2004, 281, 21-36. [CrossRef] [PubMed]

52. Fan, J.; Posner, M. Human attentional networks. Psychiatr. Prax. 2004, 31 (Suppl. 2), S210-S214. [CrossRef] [PubMed] 